TIFR-TH-98/05

February 1998

\title{
PROBING 4-DIMENSIONAL NONSUPERSYMMETRIC BLACK HOLES CARRYING D0- AND D6-BRANE CHARGES
}

\author{
Avinash Dhar* and Gautam Mandal ${ }^{\dagger}$ \\ Department of Theoretical Physics \\ Tata Institute of Fundamental Research, \\ Homi Bhabha Road, Mumbai 400 005, INDIA.
}

\begin{abstract}
We discuss a 4-dimensional nonsupersymmetric black hole solution to low energy type IIA string theory which carries D0- and D6-brane charges. For equal charges this solution reduces to the one discussed recently by Sheinblatt. We present a new parametrization of the solution in terms of four numbers which reveals the underlying brane and antibrane structure of the black hole arbitrarily far from extremality. In this parametrization, the entropy of the general nonextremal black hole takes on a simple U-duality invariant form. A Yang-Mills solution for the brane configuration corresponding to the extremal case is constructed and a computation of the 1-loop matrix theory potential for the scattering of a 0-brane probe off this brane configuration done. We find that this agrees with the 1-loop potential obtained from a supergravity calculation in the limit in which the ratio of the 0-brane to 6-brane charges is large.

*adhar@theory.tifr.res.in

† mandal@theory.tifr.res.in
\end{abstract}




\section{INTRODUCTION}

In recent years spectacular progress has been made towards a microscopic derivation of black hole thermodynamics based on string theory models [1, 2, 3, 4, 5, 6, 7, 8]. 1 Central to this development has been the existence of Dirichlet p-brane solitons (Dp-branes) of string theory, and a description of their dynamics in terms of open strings. For recent reviews of this area we refer the reader to [10, 11]. One of the remarkable and unexpected consequences of this activity has been the uncovering of a deep and potentially far-reaching connection between supergravity and superYang-Mills theories [14, 12, 13]. A precise formulation of this connection exists in the form of the matrix theory conjecture [15. Although there are several issues connected with this conjecture that need better understanding, there is an impressive body of evidence in support of it. For recent reviews in this area we refer the reader to [16, 11].

In the context of black hole physics, there have been many studies which explore this supergravity-superYang-Mills connection. Many of these studies use D-branes to probe black hole physics [17, 18, 19, 20, 21] because slowly moving D-branes act as localized probes and so can be used for big black holes, where weak coupling perturbation theory around the classical supergravity solution is good, as well as for small size ( $<$ string length, $\left.l_{s}\right)$ black holes, where perturbative D-brane gauge theory is good. In all the known cases agreement is found at 1-loop level between the supergravity and superYang-Mills calculations.

All the examples of black holes quoted above are either extremal BPS or near extremal BPS. In these cases there is either some residual supersymmetry or there is a small parameter which controls deviations from a configuration that preserves some supersymmetry. One might argue that it is this fact that is responsible for the agreement mentioned above as well as the agreement of other physical quantities like entropy, etc.

It is clearly of interest to ask what happens in the case of nonsupersymmetric black holes, i.e. those that neither preserve any supersymmetry nor are in any obvious sense close to one that does. The matrix theory conjecture requires agreement between supergravity and superYang-Mills calcu-

\footnotetext{
${ }^{1}$ For a recent review and a more complete list of references see 9 .

${ }^{2}$ Similar agreement at 1-loop level has also been found for D-brane scattering off other supersymmetric or nearly supersymmetric bound states of D-branes [23].
} 
lations even in these cases.[? It is important to verify if the conjecture is right in these cases also, since this would provide additional information about strongly coupled dynamics that does not entirely rely on supersymmetry. One such nonsupersymmetric extremal black hole solution to the classical low energy equations of Type IIA theory compactified down to 4dimensions has recently been discussed by several authors [34, 35, 36, 37]. This 4-dimensional black hole carries D0- and D6-brane charges and a qualitative microscopic picture of it as a bound state of D0- and D6-branes has been developed in [34]. The solution given in this work is, however, restricted to the case in which the D0-brane charge is equal to the D6-brane charge. In this solution the D0-brane charge cannot be varied independently and so one cannot go to the infinite momentum frame, as required by the matrix theory conjecture. Thus, for comparison with matrix theory we need to generalize the above known solution to the one in which the D0- and D6-brane charges can be varied independently.

In this paper we will discuss this generalization and compare the supergravity and superYang-Mills calculations of the effective potential at 1-loop for a D0-brane probe scattering off the black hole. The black hole solution is discussed in Sec. 2. A paramerization of the solution in terms of four numbers is presented in Sec. 3. In terms of these numbers the entropy of the black hole takes on a very simple form, even far away from extremality, revealing an underlying brane and antibrane structure. In Sec. 4 we study a slowly moving D0-brane probe in the presence of the extremal black hole background from the low energy classical closed string point of view. In

\footnotetext{
${ }^{3}$ Agreement has been shown for the nonsupersymmetric configuration of a membrane and anti-membrane [24]. The authors of this work have argued that in the limit of large boosts, required by the matrix theory conjecture, this nonsupersymmetric configuration comes close to being a supersymmetric one.

${ }^{4}$ Other examples of agreement exist. For a certain class of black holes far from extremality microscopic counting agrees with the Bekenstein-Hawking formula [25]. Microscopic counting for a nonsupersymmetric extremal black hole in Type IA theory also reproduces the Bekenstein-Hawking entropy exactly [26]. Similar agreement has been seen for entropy at the stretched horizon in the case of some nonsupersymmetric extremal elementary black holes [27]. Also, recently there has been a lot of activity in identifying Schwarzschild black holes in the matrix theory [28, 29, 30, 31, 32, 33.

${ }^{5}$ See, however, reference 36 in which a matrix theory calculation in the related problem of scattering of probe D6-branes carrying large D0-brane charge off a target D0-brane shows agreement with supergravity calculation at 1-loop.
} 
Sec. 5 we generalize the Yang-Mills construction of bound state of D0- and D6-branes of Taylor [35] to the present solution. The 1-loop matrix theory calculation is done in Sec. 6 and compared with the supergravity calculation of Sec. 4. We conclude with some remarks in Sec. 7.

After the first version of this work was submitted we learnt of a related work [38] with which we have some overlap.

\section{THE BLACK HOLE SOLUTION}

The bosonic part of the low energy effective action of Type IIA string theory is

$$
S_{10}=\frac{1}{(2 \pi)^{7} g^{2}} \int d^{10} x \sqrt{-G_{10}}\left[e^{-2 \phi}\left(R_{10}+4(\nabla \phi)^{2}\right)-\frac{1}{4} F_{10}^{2}\right]
$$

We have set all the matter terms, except the 2-form Ramond-Ramond field strength $F_{10}$, to zero. The string coupling $g$ is defined such that the dilaton field $\phi \rightarrow 0$ at spatial infinity. Also, we have used the signature $(-,+,+, \cdots)$ and string units $\sqrt{\alpha^{\prime}}=l_{s}=1$. The 10-dimensional Newton's constant is then given by $8 \pi^{6} g^{2}$ so that the overall constant in front in the action (2.1) is $1 / 16 \pi$ (Newton's constant). As discussed in [39], a solution to the classical equations of motion of the action (2.1) can be obtained from a solution to the classical equations of motion of the bosonic part of 11-dimensional supergravity action

$$
S_{11}=\frac{1}{(2 \pi)^{8} g^{3}} \int d^{11} x \sqrt{-G_{11}} R_{11}
$$

of the form

$$
d s_{11}^{2}=e^{4 \phi / 3}\left(d x_{11}+A_{\mu} d x^{\mu}\right)^{2}+e^{-2 \phi / 3} d s_{10}^{2},
$$

by compactifying $x_{11}$ on a circle of radius $g$, provided $\partial / \partial x_{11}$ is a Killing vector of the solution. The solution for the various fields can then be readoff from the from of the 11-dimensional line element in (2.3). Here $A_{\mu}$ is 10 dimensional Ramond-Ramond gauge potential from which the field strength $F_{10}$ is derived.

We are interested in a 4-dimensional solution of the 11-dimensional theory of the form (2.3) with

$$
d s_{10}^{2}=d s_{4}^{2}+e^{2 \phi / 3} \sum_{i=4}^{9} d y^{i} d y^{i}
$$


where $y_{i}, i=4,5, \cdots, 9$ are flat directions which will be compactified on a six-torus of volume $V_{6}$. Plugging (2.4) into the action (2.1) gives

$$
S_{10}=\frac{1}{2 \pi g^{2}} \frac{V_{6}}{(2 \pi)^{6}} \int d^{4} x \sqrt{-G_{4}}\left[R_{4}-\frac{2}{3}(\nabla \phi)^{2}-\frac{1}{4} e^{2 \phi} F_{4}^{2}\right]
$$

Apart from some rescalings of the dilaton and the gauge potential, and the different signature of the metric, this is exactly the action whose solutions have been derived in [40, 41, 42]. These solutions have been further discussed in different contexts in [43, 44, 45]. So we may essentially read off the desired solution from these works. For our purposes here we will closely follow [43].

The general spherically symmetric, asymptotically flat and time-independent black hole solution obtained in this way involves three arbitrary parameters, apart from the volume $V_{6}$ of the compact 6 -dimensional torus. These may be taken to be the total Ramond-Ramond "electric" charge $Q$, the corresponding "magnetic" charge $P$ and the ADM mass $M$ of the black hole. In terms of these parameters the solution may be written as

$$
\begin{aligned}
d s_{4}^{2} & =-H_{3}\left(H_{1} H_{2}\right)^{-\frac{1}{2}} d t^{2}+\left(H_{1} H_{2}\right)^{\frac{1}{2}} H_{3}^{-1} d r^{2}+\left(H_{1} H_{2}\right)^{\frac{1}{2}} d \Omega^{2}, \\
A_{\mu} d x^{\mu} & =-2\left[\frac{Q}{H_{2}}\left(r-\frac{\lambda}{3}\right) d t+P(1-\cos \theta) d \hat{\phi}\right], \\
e^{4 \phi / 3} & =H_{2} / H_{1},
\end{aligned}
$$

where $H_{1}, H_{2}, H_{3}$ are the following functions of $r$ :

$$
\begin{aligned}
& H_{1}(r)=(r-\lambda / 3)^{2}-\frac{2 \lambda P^{2}}{\lambda-3 G_{N} M}, \\
& H_{2}(r)=(r+\lambda / 3)^{2}-\frac{2 \lambda Q^{2}}{\lambda+3 G_{N} M}, \\
& H_{3}(r)=\left(r-G_{N} M\right)^{2}-\left(G_{N}^{2} M^{2}-P^{2}-Q^{2}+\lambda^{2} / 3\right) .
\end{aligned}
$$

In the above $G_{N} \equiv 8 \pi^{6} g^{2} / V_{6}$ is the 4-dimensional Newton's constant and the parameter $\lambda$ is the "dilaton charge" defined by the asymptotic behaviour

$$
\phi \rightarrow \frac{\lambda}{r}+0\left(\frac{1}{r^{2}}\right), \quad r \rightarrow \infty
$$


The parameter $\lambda$ is not independent, but depends on $Q, P$ and $M$ through the constraint

$$
\frac{Q^{2}}{\lambda+3 G_{N} M}+\frac{P^{2}}{\lambda-3 G_{N} M}=\frac{2}{9} \lambda .
$$

There exists a more convenient parametrization [40] which uses three independent parameters, $q, p$ and $a$, in terms of which the constraint (2.12) is automatically satisfied. The physical parameters $Q, P$ and $M$ are related to these by

$$
\begin{aligned}
G_{N} M & =\frac{1}{4}(q+p), \quad \lambda=\frac{3}{4}(q-p), \\
Q^{2} & =\frac{q}{4}\left(\frac{q^{2}-a^{2}}{q+p}\right), \quad P^{2}=\frac{p}{4}\left(\frac{p^{2}-a^{2}}{q+p}\right) .
\end{aligned}
$$

The inner and outer horizons of the solution are defined by the zeroes of $H_{3}(r)$. These occur at $r=r_{ \pm}$, given by

$$
\begin{aligned}
r_{ \pm} & =G_{N} M \pm\left(G_{N}^{2} M^{2}-P^{2}-Q^{2}+\lambda^{2} / 3\right)^{1 / 2} \\
& =\frac{1}{4}(q+p) \pm \frac{a}{2}
\end{aligned}
$$

We see that $a$ parametrizes devation from extremality.

The entropy, $S$, and the Hawking temperature, $T_{H}$, of the black hole are given by

$$
\begin{aligned}
S & =\frac{\pi}{G_{N}}\left(H_{1}\left(r_{+}\right) H_{2}\left(r_{+}\right)\right)^{1 / 2}=\frac{\pi}{2 G_{N}}(p q)^{1 / 2} \frac{(p+a)(q+a)}{(p+q)} \\
T_{H} & =\frac{1}{4 \pi}\left(r_{+}-r_{-}\right)\left(H_{1}\left(r_{+}\right) H_{2}\left(r_{+}\right)\right)^{-1 / 2}=\frac{a}{2 \pi}(p q)^{-1 / 2}\left(\frac{(p+a)(q+a)}{(p+q)}\right)^{-1}
\end{aligned}
$$

The geometry, thermodynamics and other properties of this solution have been extensively discussed in [43] to which we refer the reader for details. Here we will only briefly discuss two special cases. 
(i) Reissner-Nordström Solution

This requires a constant dilaton which is obtained for $\lambda=0$. The constraint (2.12) (alternatively, the parametrization (2.13)) then requires $Q=P$, i.e. in this case the solution requires the "electric" and "magnetic" charges to be equal. The ADM mass of the corresponding extremal solution is given by $G_{N} M=\sqrt{2} Q=\sqrt{2} P$. This is the solution discussed in [34. As we shall see later, comparison with matrix theory requires the ratio of electric to magnetic charges, $Q / P$, to be large. Such a comparison is clearly not possible for the Reissner-Nordström case.

(ii) The general extremal solution

This is obtained by setting $r_{+}=r_{-}$. In the parametrization (2.13) this implies that the parameter $a$ vanishes for the extremal case, as is obvious from the second equality of (2.14). Using $a=0$ in (2.13) one may solve for $q, p$ and $M$ in terms of the two independent parameters $Q$ and $P$. For the ADM mass of the extremal black hole one gets

$$
G_{N} M_{\mathrm{ext}}=\frac{1}{2}\left(Q^{2 / 3}+P^{2 / 3}\right)^{3 / 2} .
$$

Similarly, from (2.15) one gets

$$
S_{\mathrm{ext}}=\frac{2 \pi}{G_{N}} Q P
$$

Now, in terms of the integer normalized D0-brane and D6-brane charges, $Q_{0}$ and $Q_{6}$, corresponding respectively to $Q$ and $P$, we have 34

$$
Q=\frac{Q_{0}}{4 M_{6}}=2 G_{N} M_{0} Q_{0}, \quad P=\frac{Q_{6}}{4 M_{0}}=2 G_{N} M_{6} Q_{6},
$$

where

$$
M_{6}=\frac{1}{g} \frac{V_{6}}{(2 \pi)^{6}}, \quad M_{0}=\frac{1}{g},
$$

are respectively the mass of a single D6-brane and a single D0-brane. Rewriting (2.17) and (2.18) in terms of $Q_{0}$ and $Q_{6}$, we get

$$
\begin{aligned}
M_{\text {ext }} & =\left\{\left(Q_{0} M_{0}\right)^{2 / 3}+\left(Q_{6} M_{6}\right)^{2 / 3}\right\}^{3 / 2}, \\
S_{\text {ext }} & =\pi Q_{0} Q_{6} .
\end{aligned}
$$


It follows from (2.21) that the ADM mass of the extremal black hole is greater than the sum of masses of $Q_{0}$ D0-branes and $Q_{6}$ D6-branes. So it is unstable against decay into infinitely separated branes. As discussed in [46], even extreme black holes, which have a zero Hawking temperature, can decay in theories in which there exist particles with charges greater than their masses. This is so in the present case since, in 4-dimensional Planck units, both types of branes have charges which are twice their respective masses, as can be seen from (2.19). Now, for large values of $Q_{0}\left(Q_{6}\right)$, a WKB estimate for the rate of decay [46] by the emission of D0-branes (D6-branes) is $e^{-k Q_{0}}\left(e^{-k Q_{6}}\right)$, where $k$ is a constant of order unity. Hence black holes of this type with large values of the two charges are long-lived states.

A microscopic picture of this extremal black hole in terms of a bound state of $Q_{0}$ D0- and $Q_{6}$ D6-branes has been discussed in [34]. As argued there, the degrees of freedom responsible for the entropy of the black hole are the fermionic modes in the Ramond sector of the 0-6 strings. There modes are massless when the D0-brane is sitting on top of the D6-brane. Using there modes a picture of the bound state has been built and a counting of the microscopic states of the bound system done in [34. Upto a constant of order unity, the logarithm of the degeneracy of microstates is identical to the entropy of the black hole given by (2.22).

\section{MICROSCOPIC STRUCTURE OF THE GENERAL BLACK HOLE SOLUTION}

In this section we will present a new parametrization of the general black hole solution in terms of four numbers $Q_{0}, \bar{Q}_{0}, Q_{6}$ and $\bar{Q}_{6}$ which we shall trade for the four parameters $Q, P, M$ and $V_{6}$. As we shall see, in terms of these numbers the expression for the entropy of the black hole takes the very simple form

$$
S=\pi\left(Q_{0}+\bar{Q}_{0}\right)\left(Q_{6}+\bar{Q}_{6}\right)
$$

which is valid arbitrarily far from extremality and is suggestive of an underlying microscopic brane and antibrane structure. 
We begin by introducing the boost parameters $\alpha$ and $\beta$ as follows: $[9$

$$
q=a \cosh \alpha, \quad p=a \cosh \beta
$$

From (2.13) we then see that

$$
\begin{aligned}
& Q=\frac{a}{2}\left(1+\frac{\cosh \beta}{\cosh \alpha}\right)^{-1 / 2} \sinh \alpha \\
& P=\frac{a}{2}\left(1+\frac{\cosh \alpha}{\cosh \beta}\right)^{-1 / 2} \sinh \beta
\end{aligned}
$$

Now, analogous to (2.19) we wish to write (3.3) and (3.4) in the form

$$
Q=2 G_{N} M_{0}\left(Q_{0}-\bar{Q}_{0}\right), \quad P=2 G_{N} M_{0}\left(Q_{6}-\bar{Q}_{6}\right) .
$$

This suggests that we define the four numbers $Q_{0}, \bar{Q}_{0}, Q_{6}$ and $\bar{Q}_{6}$ as follows:

$$
\begin{aligned}
Q_{0} & =\frac{a}{4 G_{N} M_{0}}\left(1+\frac{\cosh \beta}{\cosh \alpha}\right)^{-1 / 2}\left(\frac{e^{\alpha}+c}{2}\right) \\
\bar{Q}_{0} & =\frac{a}{4 G_{N} M_{0}}\left(1+\frac{\cosh \beta}{\cosh \alpha}\right)^{-1 / 2}\left(\frac{e^{-\alpha}+c}{2}\right) \\
Q_{6} & =\frac{a}{4 G_{N} M_{6}}\left(1+\frac{\cosh \alpha}{\cosh \beta}\right)^{-1 / 2}\left(\frac{e^{\beta}+d}{2}\right) \\
\bar{Q}_{6} & =\frac{a}{4 G_{N} M_{6}}\left(1+\frac{\cosh \alpha}{\cosh \beta}\right)^{-1 / 2}\left(\frac{e^{-\beta}+d}{2}\right)
\end{aligned}
$$

where at this stage $c$ and $d$ are arbitrary. These are partly fixed by the requirement that the entropy of the extremal black hole, (2.22), be correctly reproduced by the above parametrization. This gives $c=d=1$ in the extremal limit. We will make this simple choice for $c$ and $d$ even away from extremality. Substituting (3.6) - (3.9) for $c=d=1$ in (2.15) then gives (3.1) for the entropy of the general nonextremal black hole.

\footnotetext{
${ }^{6}$ The parametrization $(2.13)$ of the general black hole solution restricts the sum $(p+q)$ to be positive. This allows for one of these two parameters to be negative. The following parametrization corresponds to the case in which $q$ and $p$ are both positive.
} 
We can also express the mass $M$ of the black hole and the volume $V_{6}$ of the internal $T^{6}$ in terms of the numbers $Q_{0}, \bar{Q}_{0}, Q_{6}$ and $\bar{Q}_{6}$. Using (3.6)(3.9) with $c=d=1$, we get

$$
\begin{gathered}
M=\left[\left(M_{0} \frac{Q_{0}^{2}+\bar{Q}_{0}^{2}}{Q_{0}+\bar{Q}_{0}}\right)^{2 / 3}+\left(M_{6} \frac{Q_{6}^{2}+\bar{Q}_{6}^{2}}{Q_{6}+\bar{Q}_{6}}\right)^{2 / 3}\right]^{3 / 2}, \\
\frac{M_{6}}{M_{0}}=\frac{V_{6}}{(2 \pi)^{6}}=\left[\frac{Q_{0} \bar{Q}_{0}}{Q_{6} \bar{Q}_{6}} \frac{Q_{6}^{2}+\bar{Q}_{6}^{2}}{Q_{0}^{2}+\bar{Q}_{0}^{2}}\right]^{3 / 2}\left[\frac{Q_{0}^{2}+\bar{Q}_{0}^{2}}{Q_{0}+\bar{Q}_{0}}\right]\left[\frac{Q_{6}^{2}+\bar{Q}_{6}^{2}}{Q_{6}+\bar{Q}_{6}}\right]^{-1} .
\end{gathered}
$$

For completeness we also give below the expressions for the nonextremality parameter, $a$, and the Hawking temperature, $T_{H}$ :

$$
\begin{gathered}
a=\frac{1}{M_{6}} \frac{Q_{0} \bar{Q}_{0}}{Q_{0}+\bar{Q}_{0}}\left[1+\frac{Q_{0} \bar{Q}_{0}}{Q_{6} \bar{Q}_{6}} \frac{Q_{6}^{2}+\bar{Q}_{6}^{2}}{Q_{0}^{2}+\bar{Q}_{0}^{2}}\right]^{1 / 2}, \\
T_{H}=\frac{2 M_{0}}{\pi} \frac{Q_{0} \bar{Q}_{0}}{\left(Q_{0}+\bar{Q}_{0}\right)^{2}\left(Q_{6}+\bar{Q}_{6}\right)}\left[1+\frac{Q_{0} \bar{Q}_{0}}{Q_{6} \bar{Q}_{6}} \frac{Q_{6}^{2}+\bar{Q}_{6}^{2}}{Q_{0}^{2}+\bar{Q}_{0}^{2}}\right]^{1 / 2} .
\end{gathered}
$$

The extremal limit discussed in the previous section is obtained for $\bar{Q}_{0} \rightarrow 0$ and $\bar{Q}_{6} \rightarrow 0$.

The mass formula (3.10) generalizes (2.21) to the nonextremal case and apparently corresponds to a collection of interacting branes and antibranes. Unlike the extremal case, however, the mass of a nonextremal black hole is not necessarily more than the sum of the masses of its constituent branes and antibranes. In fact, for fixed $Q$ and $P$, as we move away from extremality by adding branes and antibranes, the mass of the black hole increases at a much slower rate than the sum of masses of its constituents. This indicates that the binding becomes tighter as more and more antibranes are added to the system of branes that constitutes the extremal black hole. An extreme example of this is the neutral Schwarzschild black hole. It can be easily seen from (3.10) that the mass of Schwarzschild black hole is a factor of $1 / \sqrt{2}$ smaller than the sum of masses of its constituents. The Schwarzschild black hole, therefore, appears to be a truly bound state of its constituent branes and antibranes, unlike the extremal black hole. It Hawking decays,

\footnotetext{
${ }^{7}$ Other extremal limits can be obtained by letting any one of the pairs of charges $\left(Q_{0}, Q_{6}\right),\left(Q_{0}, \bar{Q}_{6}\right)$ and $\left(\bar{Q}_{0}, Q_{6}\right)$ vanish.
} 
however, because a brane and the corresponding antibrane can annihilate when they overlap. This decay can be neglected in the classical limit for large black holes. In the microscopic picture this seems to indicate that for large black holes the probability for branes (and antibranes) to overlap is small, presumably because the average separation between branes (and antibranes) grows with the size of the black hole.

To end this section we mention that the formulae (3.1), (3.10) and (3.13) respectively for entropy, mass and the Hawking temperature of the black hole are invariant under the conjectured U-duality group, $E_{7(7)}(\mathbf{Z})$, for the string theory under discussion [47]. This follows from the fact that the charges we are considering are inert under S-duality and are interchanged by T-duality. Moreover, the latter also interchanges $M_{0}$ and $M_{6}$. Using this and (3.11) one can then easily see the duality invarince of the entropy, mass and Hawking temperature.

\section{DO-BRANE PROBE IN THE EXTREMAL BLACK HOLE BACKGROUND}

In this section we will consider the motion of a slowly moving D0-brane probe in the presence of the extremal black hole from the point of view of classical closed string theory. This is governed by the action [10]

$$
S_{\text {Probe }}=-\frac{1}{g} \int d \tau e^{-\phi}\left|\frac{d s_{4, \mathrm{ext}}}{d \tau}\right|+\frac{1}{g} \int d \tau A_{\mu} \frac{d x^{\mu}}{d \tau}
$$

where $\tau$ parametrizes the trajectory of the probe D0-brane and $d s_{4, \text { ext }}$ refers to the line element in (2.6) specialized to the extremal case. In the static gauge, the above action becomes

$$
S_{\text {Probe }}=-\frac{1}{g} \int d t\left[K_{1}^{1 / 2} K_{2}^{-1}\left(1-K_{1} K_{2} v^{2}\right)^{1 / 2}+2 Q K_{2}^{-1} K_{3}\right]
$$

In writing (4.2) we have introduced the variable $\rho=\left(r-G_{N} M_{\text {ext }}\right)$ to bring the velocity $v$ of the probe into the standard form $v^{2}=\dot{\rho}^{2}+\rho^{2} \dot{\Omega}^{2}$. We have also dropped a term linear in velocity in the action since we will not be considering this term when we compare with the matrix theory result. The functions $K_{1}, K_{2}$ and $K_{3}$ are given by

$$
K_{1}(\rho)=1+\frac{2 P}{\rho} \sqrt{f^{2}+1}+\frac{2 P^{2}}{\rho^{2}} f^{2},
$$




$$
\begin{aligned}
& K_{2}(\rho)=1+\frac{2 P}{\rho} f^{2} \sqrt{f^{2}+1}+\frac{2 P^{2}}{\rho^{2}} f^{4}, \\
& K_{3}(\rho)=\frac{1}{\rho}\left(1+\frac{P}{\rho} \sqrt{f^{2}+1}\right),
\end{aligned}
$$

where the parameter $f$ measures the ratio of the charges

$$
f^{3} \equiv \frac{Q}{P}=\frac{Q_{0} M_{0}}{Q_{6} M_{6}}
$$

For small velocities we may expand the effective potential seen by a D0brane scattering off the black hole in powers of $v$. At large distances $\rho$, this may be further expanded in powers of $\rho$. In the present case, this latter expansion is identical to loop expansion in the string coupling. Thus, the 1-loop effective potential, correct to order $v^{4}$, is given by

$$
\begin{aligned}
V_{\text {eff }}^{(1-\text { loop })=} & -\frac{Q_{6}}{2 \rho}\left[\left\{\left(f^{2}-\frac{1}{2}\right) \sqrt{f^{2}+1}-f^{3}\right\}+\frac{3 v^{2}}{4} \sqrt{f^{2}+1}\right. \\
& \left.+\frac{v^{4}}{8}\left(f^{2}+5 / 2\right) \sqrt{f^{2}+1}+0\left(v^{6}\right)\right]
\end{aligned}
$$

Note that the black hole solution given in [34 corresponds to $f=1$. The expression for $V_{\text {eff }}^{(1-\text { loop })}$ given above agrees with that given in [37 for $f=1$ to the approximation considered there.

\section{THE YANG-MILLS SOLUTION}

It is known that both the short-range and long-range potentials between a D0-brane and a D6-brane are repulsive. It is, therefore, not possible to form a $0+6$ bound state without putting extra energy into the system. In the Yang-Mills picture this is reflected in the fact that energetically a D0-brane as an "instanton" in a D6-brane gauge theory would prefer to shrink to a point and then move away from the D6-brane. Nevertheless, as shown in [35], a Yang-Mills configuration on $T^{6}$ corresponding to 4 D6-branes and 4 D0-branes exists which is classically stable at least to quadratic order.

We now look for a Yang-Mills configuration on $T^{6}$ for generic values of the charges $Q_{0}$ and $Q_{6}$. As in [35], this may be obtained by solving the following 
equations in a $\mathcal{U}\left(Q_{6}\right)$ gauge theory:

$$
F=\eta *(F \wedge F)
$$

under the constraints

$$
\int_{2-\text { cycle }} \operatorname{Tr} F=0, \quad \int_{4-\text { cycle }} \operatorname{Tr}(F \wedge F)=0
$$

and

$$
Q_{0}=\frac{1}{6(2 \pi)^{6}} \int_{T^{6}} \operatorname{Tr}(F \wedge F \wedge F)
$$

Here $F$ is the 2-form field strength on $T^{6}$ and $*$ in (5.1) denotes Hodge dual. Also, $\eta$ is a Lagrange multiplier which forces the constraint (5.3). This constraint ensures that the configuration has $Q_{0} \mathrm{D} 0$-brane charge. The other constraints, (5.2), ensure that the configuration has vanishing D2- and D4-brane charges.

As in 35] we choose a solution with constant field strength with only the following nonvanishing components in $T^{6}$ :

$$
F_{45}=\gamma \nu_{1}, \quad F_{67}=\gamma \nu_{2}, \quad F_{89}=\gamma \nu_{3}
$$

where $\gamma$ is an arbitrary constant and $\nu_{1}, \nu_{2}$ and $\nu_{3}$ are diagonal $Q_{6} \times Q_{6}$ matrices. According to (5.2) they must satisfy

$$
\operatorname{Tr} \nu_{i}=0, \quad \operatorname{Tr}\left(\nu_{i} \nu_{j}\right)=0(i \neq j), \quad i, j=1,2,3
$$

Moreover, the equation of motion (5.1) implies that the entries in all the three $\nu_{i}$ 's must be equal in magnitude, which we may set equal to identify, without any loss of generality, because of the presence of the parameter $\gamma$ in (5.4). The equations of motion are then satisfied if

$$
\nu_{i} \nu_{j}=\left|\epsilon_{i j k}\right| \nu_{k}
$$

A solution to (5.6) satisfying (5.5) has been presented in 35 for the smallest possible value of $Q_{6}$, which is 4 . The simplest generalization of this to arbitrary $Q_{6}$ is to just repeat the $Q_{6}=4$ solution an arbitrary number of times. That is, a solution with $Q_{6}=4 n$ is given by

$$
\begin{aligned}
& \nu_{1}=\operatorname{diag}((1,1,-1,-1),(1,1,-1,-1), \cdots, n \text { times }), \\
& \nu_{2}=\operatorname{diag}((1,-1,-1,1),(1,-1,-1,1), \cdots, n \text { times }), \\
& \nu_{3}=\operatorname{diag}((1,-1,1,-1),(1,-1,1,-1), \cdots, n \text { times }) .
\end{aligned}
$$


Note that in the above the order in which the individual entries in the diagonal matrices appear may be changed without affecting the solution, provided identical changes are made in all $\nu_{i}$ 's. Thus, the following is an equivalent solution:

$$
\begin{aligned}
& \nu_{1}=\operatorname{diag}((1,1, \cdots n \text { times }),(1,1, \cdots n \text { times }),(-1,-1, \cdots n \text { times })(-1,-1, \cdots n \text { times })), \\
& \nu_{2}=\operatorname{diag}((1,1, \cdots n \text { times }),(-1,-1, \cdots n \text { times }),(-1,-1, \cdots n \text { times })(1,1, \cdots n \text { times })), \\
& \nu_{1}=\operatorname{diag}((1,1, \cdots n \text { times }),(-1,-1, \cdots n \text { times }),(1,1, \cdots n \text { times })(-1,-1, \cdots n \text { times })) .
\end{aligned}
$$

It is this form of the solution that will be convenient for the scattering calculation done in the subsequent sections.

Just as in the case of the configuration with $Q_{6}=4$ discussed in [35], all supersymmetries are broken in the present configuration as well. Since that is the case, one expects the energy of this configuration to exceed the minimal BPS energy for the $0+6$ system. In fact, using the Born-Infeld formula for diagonal field strengths, we get for the energy of the above Yang-Mills configuration

$$
\begin{aligned}
E_{Y M} & =\frac{1}{g(2 \pi)^{6}} \int_{T^{6}} \operatorname{Tr} \sqrt{\operatorname{det}\left(\delta_{\mu \nu}+F_{\mu \nu}\right)} \\
& =M_{6} \operatorname{Tr}\left\{\left(1+F_{45}^{2}\right)\left(1+F_{67}^{2}\right)\left(1+F_{89}^{2}\right)\right\}^{1 / 2} \\
& =M_{6} Q_{6}\left(1+\gamma^{2}\right)^{3 / 2}
\end{aligned}
$$

Now, from (4.3) we deduce that the parameter $\gamma$ is related to $Q_{0}$ and $Q_{6}$ by

$$
Q_{0} M_{0}=\gamma^{3} Q_{6} M_{6}
$$

From this and (3.6) we see that $\gamma=f$. Moreover, using this in (4.9) we get

$$
E_{Y M}=\left\{\left(M_{0} Q_{0}\right)^{2 / 3}+\left(M_{6} Q_{6}\right)^{2 / 3}\right\}^{3 / 2} .
$$

This is precisely the same as the expression for $M_{\text {ext }}$ in (2.21). This result is surprising since the configuration of branes that we are considering does not preserve any supersymmetry and so one might have expected the mass 
of the bound state to get renormalized in the strong coupling region which is the region in which supergravity is the effective low energy theory. A deeper appreciation of this result could be useful in understanding aspects of strongly coupled dynamics that do not rely entirely on supersymmetry.

\section{MATRIX THEORY CALCULATION}

In this section we will calculate the 1-loop effective potential for a D0-brane probe scattering off the configuration of D0- and D6-branes constructed in the previous section. By now there exist many calculations of 1-loop effective potential of D-brane probes scattering off various configurations of D-branes within the matrix theory framework. The set-up for a 1-loop calculation of D0-brane - D6-brane scattering has been formulated in [48 and the calculation done for the special case in which the three magnetic fluxes on the D6-branes are equal. From (5.8) we see that we need this calculation for a more general case. We now proceed to do this calculation.

The only dynamical degrees of freedom in matrix theory are D0-branes and their dynamics is governed by the quantum mechanical action [15 obtained by dimensional reduction of 10-dimensional superYang-Mills action to 1-dimension:

$$
\begin{gathered}
S=\frac{1}{2 g} \int d t \operatorname{Tr}\left\{\left(D_{t} X_{i}\right)^{2}+\frac{1}{2}\left[X_{i}, X_{j}\right]^{2}-\left(\bar{D}_{t} A\right)^{2}+\theta^{T} D_{t} \theta\right. \\
\left.+i \theta^{T} \gamma^{i}\left[X_{i}, \theta\right]+2 \partial_{t} C^{\dagger} D_{t} C-2\left[C^{\dagger}, B_{i}\right]\left[X_{i}, C\right]\right\} .
\end{gathered}
$$

Here $g$ is the string coupling, $X_{i}(i=1,2, \cdots 9)$ are matrix valued space components of $A_{\mu}$ (the 10-dimensional gauge potential), $A_{0}$ is the timecomponent of $A_{\mu}, \theta$ is a real 16-component spinor $\left(\theta^{T}\right.$ is transposed in spinor indices only), $\gamma^{i}(i=1,2, \cdots 9)$ are nine real symmetric $16 \times 16$ Dirac matrices satisfying $\left(\gamma^{i}\right)^{2}=1$ and, finally, $C, C^{\dagger}$ are the ghost fields. $B_{i}$ is the background value for $X_{i}$ and we have chosen $B_{0}=0$. Also,

$$
\begin{aligned}
D_{t} X_{i} & =\partial_{t} X_{i}-i\left[A, X_{i}\right], \quad D_{t} \theta=\partial_{t} \theta-i[A, \theta], \\
D_{t} C & =\partial_{t} C-i[A, C], \quad \bar{D}_{t} A=\partial_{t} A+i\left[B_{i}, X_{i}\right] .
\end{aligned}
$$

Note that the dimensional reduction has been done after background gaugefixing and ghost terms have been added to the 10-dimensional action. 


\section{Background Configuration}

The above quantum mechanical action was studied in 49] in connection with the 11-dimensional supermembrane and in [50] in the context of Dpbranes in matrix theory formuation of M-theory. In this latter framework, all Dp-branes are made of D0-brane constituents and can be obtained as classical configurations of the action (6.1). The configuration we desire, which is expected to correspond to the extremal black hole solution of sec. 2, consists of D6-branes with the D0-branes appearing on them as magnetic fluxes. A multiple six-brane configuration with magnetic fluxes in (45), (67) and (89) directions is given by the following

$$
B_{4,6,8}=\left(\begin{array}{cc}
Q_{1,2,3} & 0 \\
0 & 0
\end{array}\right), B_{5,7,9}=\left(\begin{array}{cc}
P_{1,2,3} & 0 \\
0 & 0
\end{array}\right)
$$

where the entry in the right lower corner is a single element one. This entry is for the probe D0-brane. For a single six-brane $\left[Q_{a}, P_{a}\right]=i c_{a}, a=1,2,3$, while for multiple six-branes the $Q_{a}$ 's and $P_{a}$ 's have a further structure:

$$
Q_{a}=\left(\begin{array}{ccc}
Q_{a}^{1} & & \\
& Q_{a}^{2} & \\
& & \ddots .
\end{array}\right), P_{a}=\left(\begin{array}{ccc}
P_{a}^{1} & & \\
& P_{a}^{2} & \\
& & \ddots .
\end{array}\right)
$$

where $\left[Q_{a}^{1}, P_{a}^{1}\right]=i c_{a}^{1}$, etc. The six-branes are wrapped on a $T^{6}$ with volume $V_{6}$ which is assumed to be large since we will be neglecting the effect of winding modes. For this configuration to correspond to our extremal black hole we need the upper index on $Q_{a}$ 's and $P_{a}$ 's to run from 1 to $Q_{6}$.

Now, the configuration of magnetic fluxes in (5.8) implies that the $Q_{6}(=$ $4 n)$ D6-branes can be organised into 4 sets, each consisting of $n$ D6-branes. Each D6-brane in the first set carries magnetic fluxes $\left(F_{45}, F_{67}, F_{89}\right)=(f, f, f)$. D6-branes in the other three sets carry the fluxes $(f,-f,-f),(-f,-f, f)$ and $(-f, f,-f)$. Thus a more suitable notation for the $Q_{a}$ 's and $P_{a}$ 's is $Q_{a}^{l, \alpha}, P_{a}^{l, \alpha}$ where $l=1,2,3,4$ and $\alpha=1,2, \ldots n$ and $\left[Q_{a}^{l, \alpha}, P_{a}^{m, \beta}\right]=i \delta^{\alpha \beta} \delta^{l m} c_{a}^{l, \alpha}$. For the desired configuration we need to take $c_{a}^{l, \alpha}=c_{l}^{a}$ to be independent of $\alpha$. Moreover, the four triplets of numbers $\left\{c_{l}^{a}\right\} \equiv\left(c_{l}^{1}, c_{l}^{2}, c_{l}^{3}\right) \equiv \vec{c}_{l}$ crrespond to the four triplets of fluxes listed above and so we may write

$$
\begin{aligned}
\vec{c}_{l} & =c \vec{\epsilon}_{l}, \\
\vec{\epsilon}_{1} & =(1,1,1), \vec{\epsilon}_{2}=(1,-1,-1), \vec{\epsilon}_{3}=(-1,-1,1), \vec{\epsilon}_{4}=(-1,1,-1)(6.3)
\end{aligned}
$$


As we shall see, agreement with supergravity calculation requires $c=f^{-1} \rightarrow$ 0 .

Let us now consider a D0-brane probe scattering off this background in directions transverse to the D6-branes. This is represented by the appearance of the additional backgrounds

$$
B_{1}=\left(\begin{array}{cc}
0 & 0 \\
0 & v t
\end{array}\right), B_{2}=\left(\begin{array}{ll}
0 & 0 \\
0 & b
\end{array}\right), B_{3}=0
$$

The only nonzero entry in the above matrices is in the lower right corner. Here $v$ is the velocity of the D0-brane, assumed to be along $x^{1}$, and $b$ is the impact parameter.

$\underline{\text { Fluctuations }}$

In order to compute the effective potential for the scattering of the D0brane off the configuration of D0- and D6-branes represented by the background values $B_{i}$, we need to insert these background values in the action (6.1) and integrate out the fluctuations around the background. There are basically two types of fluctuations. The first type are nonzero square matrices which are fluctuations around $Q_{a}^{l, \alpha}$ and $P_{a}^{l, \alpha}$. These represent open strings connecting the various branes in the background configuration. However, these do not contribute to the 1-loop potential, so we will not consider them here any further. They do, however, contribute to the potential at 2-loop [51] and beyond. The other type are the ones with nonzero values in the last column or row of the matrices $X_{i}, \theta$, etc. These represent open strings connecting the probe D0-brane and the branes in the background configuration. They are the relevant fluctuations for the present calculation. We shall paramterize these fluctuations as follows. Writing $X_{i}=B_{i}+\sqrt{g} Y_{i}$, we have

$$
Y_{i}=\left(\begin{array}{cc}
0 & \phi_{i} \\
\phi_{i}^{+} & 0
\end{array}\right)
$$

Similarly,

$$
A=\sqrt{g}\left(\begin{array}{cc}
0 & \phi_{A} \\
\phi_{A}^{+} & 0
\end{array}\right), \theta=\sqrt{g}\left(\begin{array}{cc}
0 & \chi_{\theta} \\
\chi_{\theta}^{+} & 0
\end{array}\right), C=\sqrt{g}\left(\begin{array}{cc}
0 & \chi_{c} \\
\tilde{\chi}_{c}^{T} & 0
\end{array}\right) .
$$


Note that, in the notation used earlier, the fluctuations have the index structure $\phi_{\ldots}^{l, \alpha}$ and $\chi_{\ldots}^{l, \alpha}$. It is also useful to paramterize the background as

$$
B_{i}=\left(\begin{array}{cc}
D_{i} & 0 \\
0 & \\
0
\end{array}\right)
$$

\section{Action for fluctuations}

We may now expand the action (6.1) around the background $B_{i}$. The terms linear in fluctuations vanish because $B_{i}$ is a solution of equations of motion. For a 1-loop calculation of the effective potential it is sufficient to retain only the quadratic terms in the fluctuations. The action for fluctuations is then given by the sum of the following four pieces:

$$
\begin{aligned}
S_{Y}= & \sum_{l, \alpha} \int d \tau\left[\phi_{i}^{l, \alpha^{\dagger}}\left(2 H_{\tau}+2 H^{l, \alpha}+b^{2}\right) \phi_{i}^{l, \alpha}\right. \\
& +2 i c_{l}^{1}\left(\phi_{4}^{l, \alpha^{\dagger}} \phi_{5}^{l, \alpha}-\phi_{5}^{l, \alpha^{\dagger}} \phi_{4}^{l, \alpha}\right) \\
& +2 i c_{l}^{2}\left(\phi_{6}^{l, \alpha^{\dagger}} \phi_{7}^{l, \alpha}-\phi_{7}^{l, \alpha^{\dagger}} \phi_{6}^{l, \alpha}\right) \\
& \left.\quad+2 i c_{l}^{3}\left(\phi_{8}^{l, \alpha^{\dagger}} \phi_{9}^{l, \alpha}-\phi_{9}^{l, \alpha^{\dagger}} \phi_{8}^{l, \alpha}\right)\right] \\
S_{A}= & \sum_{l, \alpha} \int d \tau\left[\phi_{A}^{l, \alpha^{\dagger}}\left(2 H_{\tau}+2 H^{l, \alpha}+b^{2}\right) \phi_{A}^{l, \alpha}+2 i v_{E}\left(\phi_{1}^{l, \alpha^{\dagger}} Q_{A}^{l, \alpha}-\phi_{A}^{l, \alpha^{\dagger}} \phi_{1}^{l, \alpha}\right)\right] \\
S_{\theta}= & i \sum_{l, \alpha} \int d \tau\left[\chi_{\theta}^{l, \alpha^{\dagger}}\left(\partial_{\tau}-\gamma^{i}\left(D_{i}^{l, \alpha}-d_{i}^{l, \alpha}\right)\right) \chi_{\theta}^{l, \alpha}\right], \\
S_{c}= & \sum_{l, \alpha} \int d \tau\left[\phi_{c}^{l, \alpha^{\dagger}}\left(2 H_{\tau}+2 H^{l, \alpha}+b^{2}\right) \phi_{c}^{l, \alpha}+\tilde{\phi}_{c}^{l, \alpha^{\dagger}}\left(2 H_{\tau}+2 H^{l, \alpha}+b^{2}\right) \tilde{\phi}_{c}^{l, \alpha}\right] .
\end{aligned}
$$

In writing the above, we have already made the Wick rotation $t \rightarrow i \tau, A \rightarrow$ $-i A$ and $v_{t} \rightarrow v_{E} \tau, v_{E}=i v$, since calculations are more conveniently done in Euclidean space. Also,

$$
\begin{aligned}
H_{\tau} & =\frac{1}{2}\left(-\partial_{\tau}^{2}+v_{E}^{2} \tau^{2}\right) \\
H^{l, \alpha} & =\frac{1}{2} \sum_{a=1}^{3}\left[\left(P_{a}^{l, \alpha}\right)^{2}+\left(Q_{a}^{l, \alpha}\right)^{2}\right] .
\end{aligned}
$$


Integrating out the various fluctuations gives a product of determinants. The ghost determinants cancel against those coming from $\phi_{2}^{l, \alpha}$ and $\phi_{3}^{l, \alpha}$ sectors. The remaining determinants in the bosonic sector involve the following operators (obtained after diagonalizing in the $\left(A, \phi_{1}\right)$ and $\left(\phi_{4}, \phi_{5}\right),\left(\phi_{6}, \phi_{7}\right)$ and $\left(\phi_{8}, \phi_{9}\right)$ sectors $)$ :

$$
\begin{aligned}
O_{\tau \pm}^{l, \alpha} & \equiv 2 H_{\tau}+2 H^{l, \alpha}+b^{2} \pm 2 v_{E} \longrightarrow \frac{1}{\sqrt{2}}\left(\phi_{1}^{l, \alpha} \pm i \phi_{A}^{l, \alpha}\right) \\
O_{1 \pm}^{l \alpha} & \equiv 2 H_{\tau}+2 H^{l, \alpha}+b^{2} \pm 2 c_{l}^{2} \longrightarrow \frac{1}{\sqrt{2}}\left(\phi_{4}^{l, \alpha} \pm i \phi_{5}^{l, \alpha}\right) \\
O_{2 \pm}^{l, \alpha} & \equiv 2 H_{\tau}+2 H^{l, \alpha}+b^{2} \pm 2 c_{l}^{2} \longrightarrow \frac{1}{\sqrt{2}}\left(\phi_{6}^{l, \alpha} \pm i \phi_{7}^{l, \alpha}\right) \\
O_{3 \pm}^{l, \alpha} & \equiv 2 H_{\tau}+2 H^{l, \alpha}+b^{2} \pm 2 c_{l}^{3} \longrightarrow \frac{1}{\sqrt{2}}\left(\phi_{8}^{l, \alpha} \pm i \phi_{9}^{l, \alpha}\right)
\end{aligned}
$$

Shown against each of these operators are the corresponding "diagonal" field combinations. Thus, the bosonic sector gives rise to the following product of determinants:

$$
\prod_{l=1}^{4} \prod_{\alpha=1}^{n}\left\{\operatorname{det} O_{\tau+}^{l, \alpha} \operatorname{det} O_{\tau-}^{l, \alpha} \prod_{a=1}^{3}\left(\operatorname{det} O_{a+}^{l, \alpha} \operatorname{det} O_{a-}^{l, \alpha}\right)\right\}^{-1}
$$

Now, all the above operators are diagonal in the oscillator number representation of the (1-dimensional) harmonic oscillator for $H_{\tau}$ and (3-dimensional) harmonic oscillator for $H^{l, \alpha}$. In this representation it is clear, because of the form of (6.3), that the eigenvalues of $H^{l, \alpha}$ do not depend on $l, \alpha$. In fact, the determinants in (6.8) depend only on $l$ and this dependence comes only from the mixing terms in the various operators. To see what this dependence on $l$ entails consider, for example, the product

$$
\prod_{l=1}^{4} \prod_{\alpha=1}^{n}\left(\operatorname{det} O_{1+}^{l, \alpha} \operatorname{det} O_{1-}^{l, \alpha}\right)^{-1}
$$

We have, from (5.3),

$$
\begin{aligned}
O_{1 \pm}^{l, \alpha} & =2 H_{\tau}+2 H^{l, \alpha}+b^{2} \pm 2 c \text { for } l=1,2 \\
& =2 H_{\tau}+2 H^{l, \alpha}+b^{2} \mp 2 c \text { for } l=3,4
\end{aligned}
$$


In other words, the two operators involving $+2 c$ and $-2 c$ are each repeated 4 times in the above product over $l$, giving

$$
\left\{\operatorname{det}\left(2 H_{\tau}+2 H+b^{2}+2 c\right) \operatorname{det}\left(2 H_{\tau}+2 H+b^{2}-2 c\right)\right\}^{-Q_{6}},
$$

where we have used $4 n=Q_{6}$. We have also used the fact that the eigenvalues of $H^{l, \alpha}$ do not depend on $l, \alpha$ and, dropped these indices on $H$. In this way one can show that the product of determinants in (6.9) is equal to

$$
\begin{aligned}
& \left\{\operatorname{det}\left(2 H_{\tau}+2 H+b^{2}+2 v_{E}\right) \operatorname{det}\left(2 H_{\tau}+2 H+b^{2}-2 v_{E}\right)\right. \\
& \left.\times\left(\operatorname{det}\left(2 H_{\tau}+2 H+b^{2}+2 c\right) \operatorname{det}\left(2 H_{\tau}+2 H+b^{2}-2 c\right)\right)^{3}\right\}^{-Q_{6}}
\end{aligned}
$$

To evaluate the fermionic determinant coming from (6.6), we first "square" the operator involved using the fact that $\gamma^{3}$ does not appear in the operator $i\left(\partial_{\tau}-\gamma^{i}\left(D_{i}^{l, \alpha}-d_{i}^{l, \alpha}\right)\right)$. This gives the operator $\left(2 H_{\tau}+2 H^{l, \alpha}+b^{2}+v_{E} \gamma^{1}+\right.$ $\Sigma_{a=1}^{3} c_{l}^{a} \sigma^{a}$ ) where $\sigma^{1}=i \gamma^{4} \gamma^{5}, \sigma^{2}=i \gamma^{6} \gamma^{7}$ and $\sigma^{3}=i \gamma^{8} \gamma^{9}$. This operator involves mixing coming from the $\gamma$-matrix term $\left(v_{E} \gamma^{1}+\vec{c}_{l} \cdot \vec{\sigma}\right)$. It can be diagonalised and its eigenvalues are

$$
\begin{aligned}
& \pm c\left(\epsilon_{l}^{1}+\epsilon_{l}^{2}+\epsilon_{l}^{3}\right) \pm v_{E}, \pm c\left(\epsilon_{l}^{1}+\epsilon_{l}^{2}-\epsilon_{l}^{3}\right) \pm v_{E} \\
& \pm c\left(\epsilon_{l}^{1}-\epsilon_{l}^{2}+\epsilon_{l}^{3}\right) \pm v_{E}, \pm c\left(-\epsilon_{l}^{1}+\epsilon_{l}^{2}+\epsilon_{l}^{3}\right) \pm v_{E}
\end{aligned}
$$

where all four combinations of the \pm signs are allowed in each of these, making a total of sixteen eigenvalues. Substituting the values of $\vec{\epsilon}_{l}$ from (6.3), we see that for each of the values of $l$ these eigenvalues reduce to either $\pm 3 c \pm v_{E}$ (each combination of \pm signs occuring once) or $\pm c \pm v_{E}$ (each combination of \pm signs occuring three times). Thus, the product over $l$ of the determinants in the fermionic sector also simplifies and we get for the contribution of this sector

$$
\begin{aligned}
& \left\{\left(\operatorname{det}\left(2 H_{\tau}+2 H+b^{2}+3 c+v_{E}\right) \operatorname{det}\left(2 H_{\tau}+2 H+b^{2}+3 c-v_{E}\right) \times\right.\right. \\
\times & \left.\operatorname{det}\left(2 H_{\tau}+2 H+b^{2}-3 c+v_{E}\right) \operatorname{det}\left(2 H_{\tau}+2 H+b^{2}-3 c-v_{E}\right)\right)^{1 / 2} \times \\
\times & \left(\operatorname{det}\left(2 H_{\tau}+2 H+b^{2}+c+v_{E}\right) \operatorname{det}\left(2 H_{\tau}+2 H+b^{2}+c-v_{E}\right) \times\right. \\
\times & \left.\left.\operatorname{det}\left(2 H_{\tau}+2 H+b^{2}-c+v_{E}\right) \operatorname{det}\left(2 H_{\tau}+2 H+b^{2}-c-v_{E}\right)\right)^{3 / 2}\right\}^{Q_{6}}(6.10)
\end{aligned}
$$


Note that for $v_{E}=0=c$, all the determinants in (6.9) and (6.10) become equal and these two expressions cancel against each other. This fact would seem to indicate that the present nonsupersymmetric configuration of branes becomes supersymmetric in the infinite momentum frame which corresponds to $c=f^{-1}=0$.

Apart from the power $Q_{6}$, the expressions appearing in (6.9) and (6.10) are exactly the ones that have been evaluated earlier in 48 . The result in the present case for the 1-loop long range effective potential is just $Q_{6}$ times that obtained in that work. For small $c$ and $v$, the potential correct to order $v^{4}$ is, then,

$$
V_{\text {matrix }}^{1-\text { loop }}=-\frac{Q_{6}}{\rho}\left(-\frac{3 c}{16}+\frac{3 v^{2}}{8 c}+\frac{v^{4}}{16 c^{3}}+0\left(v^{6}\right)\right)
$$

Comparing this with (4.7) we see that it agrees with the leading term in the former in the limit $f=c^{-1} \rightarrow \infty$. We also see that in this limit and at low velocities the $v^{4}$ term is the leading term (since all higher powers of $v$ are accompanied by the same factor $c^{-3}$; this is easily seen from (4.2) (4.5)). Thus we see explicitly that the scattering is dominated by D0-brane D0-brane scattering, and hence approaches a supersymmetric configuration, in this limit. In fact, assuming that this continues to be the case beyond the 1-loop term, we can use the 2-loop calculation of [22] to predict the form of the matrix theory 2-loop potential in the present case. According to the calculation of [22] the $v^{4}$ term in the D0-brane - D0-brane scattering does not get renormalized at the 2-loop level. For the present case of a D0-brane scattering off the extremal black hole this implies that at the 2-loop level, in the limit $f=c^{-1} \rightarrow \infty$, the largest power of $f$, in the low-velocity expansion, can only come with a term of order $v^{6}$ or higher power of $v$. Using (4.2) (4.5), we can obtain the 2-loop effective supergravity potential:

$$
\begin{aligned}
V_{\text {eff }}^{2-\text { loop }}=\frac{g Q_{6}^{2}}{4 \rho^{2}}\left[\left\{f^{6}-f^{3}\left(f^{2}-\frac{1}{2}\right) \sqrt{f^{2}+1}-\frac{3 f^{2}+1}{8}\right\}-\frac{3 v^{2}}{16}\left(3 f^{2}+1\right)\right. \\
-\frac{3 v^{4}}{32}\left\{4 f^{4}+\frac{5}{2}\left(3 f^{2}+1\right)\right\}-\frac{v^{6}}{64}\left\{4 f^{6}+36 f^{4}\right. \\
\left.\left.+\frac{35}{2}\left(3 f^{2}+1\right)\right\}+0\left(v^{8}\right)\right]
\end{aligned}
$$


In the limit $f \rightarrow \infty$, the largest power, $f^{6}$, comes with $v^{6}$ (and this is the highest power of $f$ that comes with all the higher order terms in $v$ ). Therefore, the supergravity calculation agrees with the predicted behaviour of the 2-loop matrix theory potential!

\section{CONCLUDING REMARKS}

We have discussed a 4-dimensional nonsupersymmetric black hole solution to low energy type IIA string theory compactified on a six-torus. The solution is parametrized by three parameters which are the ADM mass and D0- and D6brane charges, in addition to the volume of the six-torus. We have presented a new parametrization of this solution which trades these four parameters for four numbers. In terms of these numbers the entropy of the general nonextremal black hole takes on a very simple U-duality invariant form which suggests an underlying brane and antibrane structure. It would be useful to develop this picture further since it is likely to yield further information about strong coupling dynamics that does not rely entirely on supersymmetry.

The extremal solution has only the two charges as independent parameters, its mass being determined in terms of these. It is unstable against decay by emission of the branes, but for large values of the charges it is long lived. In this case there is a microscopic picture as a bound state of D0- and D6-branes. We have obtained a configuration of D0- and D6-branes, which has energy equal to the ADM mass of the extremal black hole, as a classical solution in a $\mathcal{U}\left(Q_{6}\right)$ Yang-Mills theory on $T^{6}$. We considered the corresponding brane configuration in matrix theory and computed the 1-loop effective potential for the scattering of a D0-brane probe off this brane configuration. We found that this agreed with the corresponding supergravity calculation in the limit in which the ratio of the D0- to D6-brane charges is large. In fact, assuming that this continues to be true beyond 1-loop order, we used the 2-loop calculation of [22] to make a prediction for the present case. As we have seen, this prediction agrees with the corresponding 2-loop supergravity calculation. Thus, although the extremal black hole solution is not protected by supersymmetry, it would seem that an appropriate limit exists in which the underlying brane configuration approaches a supersymmetric state in a definite and calculable way.

One of the defining features of a black hole is the event horizon. A 1- 
loop matrix theory computation sees only the Newtonian potential $\sim 1 / \rho$ and misses the subleading corrections which depend on the horizon size. To see the horizon size, one needs to go beyond a 1-loop computation. A 2loop computation was done for the case of the 5-dimensional black hole in [17] and it disagrees with the supergravity prediction, but the status of this disagreement is not clear. In the present case, however, there are already some positive indications about the 2-loop term, as we have argued at the end of the last section. For these reasons it is clearly of interest to do a complete calculation of the 2-loop term [51]. 


\section{References}

[1] A. Sen, Mod. Phys. Lett. A10 (1995) 2081 (hep-th/9504147).

[2] A. Strominger and C. Vafa, Phys. Lett. B379 (1996) 99 (hepth/9601029).

[3] C. Callan and J. Maldacena, Nucl. Phys. B472 (1996) 591 (hepth/9602043).

[4] J. Maldacena and L. Susskind, Nucl. Phys. B475 (1996) 679 (hepth/9604042).

[5] A. Dhar, G. Mandal and S.R. Wadia, Phys. Lett. B388 (1996) 51 (hepth/9605234).

[6] S.R. Das and S.D. Mathur, Nucl. Phys. B478 (1996) 561 hepth/9606185).

[7] J. Maldacena and A. Strominger, Phys. Rev. D55 (1997) 861 (hepth/9609026).

[8] S.F. Hassan and S.R. Wadia, hep-th/9703163 and hep-th/9712213.

[9] J. Maldacena, Nucl. Phys. Proc. Suppl. 61A (1998) 111 (hepth/9705078).

[10] J. Polchinski, S. Chaudhuri and C.V. Johnson, hep-th/9602052; J. Polchinski, hep-th/9611050.

[11] W. Taylor, hep-th/9801182.

[12] D. Kabat and P. Pouliot, Phys. Rev. Lett. 77 (1996) 1004 (hepth/9603127).

[13] C. Bachas, Phys. Lett. B374 (1996) 37 (hep-th/9511043).

[14] M.R. Douglas, D. Kabat, P. Pouliot and S. Shenker, Nucl. Phys. B485 (1997) 85 (hep-th/9608024).

[15] T. Banks, W. Fischler, S. Shenker and L. Susskind, Phys. Rev. D55 (1997) 5112 (hep-th/9610043). 
[16] T. Banks, hep-th/9710231; D. Bigatti and L. Susskind, hepth/9712072.

[17] M. Douglas, J. Polchinski and A. strominger, hep-th/9703031.

[18] J. M. Maldacena, hep-th/9705053 and hep-th/9709099.

[19] M. Li and E. Martimec, hep-th/9703211, hep-th/9704134 and hepth/9709114.

[20] R. Dijkgraaf, E. Verlinde and H. Verlinde, hep-th/9704018 and hepth/9709087.

[21] I. Chepelev and A.A. Tseyltin, hep-th/9704127.

[22] K. Becker and M. Becker, hep-th/9705091.

[23] G. Lifschytz, Phys. Lett. B388 (1996) 720 (hep-th/9604156) and Nucl. Phys. B499 (1997) 283 (hep-th/9610125).

[24] G. Lifschytz and S.D. Mathur, Nucl. Phys. B507 (1997) 621 (hepth/9612087).

[25] G.T. Horowitz, D.A. Lowe and J.M. Maldacena, Phys. Rev. Lett. 77 (1996) 430 (hep-th/9603195).

[26] A. Dabholkar, hep-th/9702050.

[27] M. Duff, J.T. Liu and J. Rahmfeld, Nucl. Phys. B494 (1997) 161 (hepth/9612015); M. Duff and J. Rahmfeld, Nucl. Phys. B481 (1996) 332 (hep-th/9605085).

[28] T. Banks, W. Fischler, I. Klebanov and L. Susskind, hep-th/9709091.

[29] I. Klebanov and L. Susskind, hep-th/9709108.

[30] E. Halyo, hep-th/9709225.

[31] G. Horowitz and E. Martinec, hep-th/9710217.

[32] M. Li, hep-th/9710226. 
[33] T. Banks, W. Fischler, I. Klebanov and L. Susskind, hep-th/9711005.

[34] H.J. Sheinblatt, hep-th/9705054.

[35] W. Taylor, hep-th/9705116.

[36] E. Keski-Vakkuri and P. Kraus, hep-th/9706196.

[37] J.M. Pierre, hep-th/9707102.

[38] A. Brandhuber, N. Itzhaki, J. Sonnenschein and S. Yankielowicz, hepth/9711010.

[39] F. Dowker, J. Gauntlett, G. Gibbons and G. Horowitz, Phys. Rev. D53 (1996) 7115 (hep-th/9512154).

[40] P. Dobiasch and D. Maison, Gen. Relativ. Gravitation 14 (1982) 231.

[41] A. Chodos and S. Detweiler, Gen. Relativ. Gravitation 14 (1982) 879.

[42] D. Pollard, J. Phys. A16 (1983) 565.

[43] G. Gibbons and D.L. Wiltshire, Ann. Phys. 167 (1986) 201.

[44] G. Gibbons and R. Kallosh, Phys. Rev. D51 (1995) 2839.

[45] H. Lu and C. N. Pope, hep-th/9606047.

[46] G. Gibbons, Commun. Math. Phys. 44 (1975) 245.

[47] C. Hull and P. Townsend, Nucl. Phys. B438 (1995) 109 (hepth/9410167).

[48] G. Lifschytz, hep-th/9612223.

[49] B. de Wit, J. Hoppe and H. Nicolai, Nucl. Phys. B305 [FS23] (1988) 545; B. de Wit, M. Luscher and H. Nicolai, Nucl. Phys. B320 (1989) $135 ;$ hep-th/9510017.

[50] T. Banks, N. Seiberg and S. Shenker, Nucl. Phys. B490 (1997) 91 (hep-th/9612157).

[51] A. Dhar and G. Mandal, work in progress. 